\title{
31st International EuroPACS Meeting
}

President: Emanuele Neri, MD (I) 